\title{
Whole body vibration training elevates creatine kinase levels in sedentary subjects
}

\author{
Boris Gojanovic ${ }^{a}$, Francois Feihl $^{b}$, Lucas Liaudet $^{b}$, Gérald Gremion $^{a}$, Bernard Waeber $^{b}$ \\ a Sports Medicine Unit, Centre Hospitalier Universitaire Vaudois and University of Lausanne, Switzerland \\ ${ }^{\mathrm{b}}$ Clinical pathophysiology, Department of Medicine, Centre Hospitalier Universitaire Vaudois and University of Lausanne, Switzerland
}

\section{Summary}

PRINCIPLES: Whole body vibration (WBV) is an increasingly popular modality of muscle training, especially in sedentary subjects. We hypothesised that the vigorous muscle contractions elicited by WBV can cause muscle damage expressed as an elevation in muscle enzymes.

METHODS: Twenty inactive subjects, ten male and ten female, aged $22.7 \pm 2.6$, BMI $22.4 \pm 2.1$ were included based on the absence of regular physical activity as defined by international guidelines, and no history of recent trauma, musculoskeletal pathology, implanted prosthetics, cardiovascular disease or drug intake. The intervention consisted of one bout of high intensity WBV corresponding to a typical training session, involving all the major muscle groups. Plasma levels of muscle enzymes prior to and at 24, 48 and 96 hours post exercise (creatine kinase - CK, MB fraction, troponin I, aminotransferases and lactate dehydrogenase) were measured. In addition, blood lactate was assayed immediately after exercise. Delayed onset muscle soreness (DOMS) was evaluated using a visual analogical scale.

RESULTS: Five participants (25\%) showed a significant increase in post exercise CK levels ( $>$ double of baseline). Maximal value was $3520 \mathrm{U} / 1$. No change was observed in CK-MB or troponin I. Lactate increased to $10.0 \pm 2.4$ in men and $6.9 \pm 2.4$ in women. All participants had some degrees of DOMS, without correlation to enzymatic changes. DISCUSSION: WBV can provoke high CK elevation in healthy, medication-free inactive subjects. Such an elevation is transient and harmless, but could be wrongly attributed to drug induced myopathy, as in patients treated with statins. Practitioners should bear this in mind before discontinuing a potential life saving drug.

Key words: whole body vibration; exercise; muscle enzymes; statin; myopathy

\section{Introduction}

Whole body vibration (WBV) is a fairly recent training modality, commercially available since the start of the millennium. It was developed in the 1970s in the former Soviet Union, in order to train cosmonauts and prevent loss of bone mineral and muscle mass during space flights. The principle is to execute various types of movements (squatting, for example) involving muscular contractions whilst standing on a platform that moves up and down at a very high frequency (between 20 and $50 \mathrm{~Hz}$ ). These vibrations have been described as eliciting more muscle contraction than the same exercises executed without vibrations. It is nowadays widely used for personal training purposes, with numerous claims from manufacturers, encompassing enhanced muscular strength [1], bone mass [2] , proprioception, balance [3-6] , flexibility, along with beneficial metabolic effects, such as an augmented lipolysis [7], glucose tolerance [8], growth hormone and testosterone secretion [9]. Recently, improvements in overall fitness have been shown after one year of WBV training in elderly subjects [10]. The evidence of benefits is as of yet scarce, but does however point to an interesting potential, especially in inactive and older subjects.

Are there any dangers with the use of vibration training in subjects unaccustomed to regular physical activity? The importance of physical activity in maintenance or improvement of general health is widely accepted and based on the observation that the higher the fitness level, the better the outcome for health in general [11-13]. But getting people to be active is another story. Here, the use of novel training modalities can sometimes help, especially when the promise of maximal benefits in a short time is proposed. WBV attracts a lot of sedentary people, in wellness centres or at home. However, the "aggressive" muscular contraction provoked by the machines could somehow be deleterious to those muscles, if they induced significant lesions at the myofibrillar level, or might even be of some danger in patients with comorbidities, such as diabetes, renal failure or those under medication known to affect the muscles, such as statins.

Muscular lesions are common after intensive exercise and can lead to rhabdomyolysis, [14] with severe elevation of creatine kinase (CK). In healthy subjects, both long endurance events [15] (marathon running, triathlons) and short duration heavy eccentric-type muscle contractions [16] (running/walking downhill) are known to elevate CK above $10^{\prime} 000 \mathrm{U} / 1$, even up to 100 '000 U/1, which represents a level of rhabdomyolysis potentially associated with 
renal dysfunction, if hydration levels are inadequate or acidosis is present at the same time. This urged us to assess whether WBV may also lead to muscle damage in sedentary subjects, as reflected by increased CK levels, a question that has not yet been addressed in the literature to our knowledge.

\section{Methods}

\section{Subjects}

Twenty volunteers, ten inactive males and ten inactive females were recruited among the general population by public announcement. They were identified as inactive according to the minimal activity guidelines released by the American College of Sports Medicine (less than thirty minutes of moderate physical activity five times a week), and by a standardized questionnaire (IPAQ) [17]. Some of them had been actively involved in sports in the past, but not in the last 12 months. Exclusion criteria included any of the following: epilepsy, fracture in the preceding six months, any implanted prosthetics, thrombosis in the preceding three months, history of diabetes, cardiovascular pathology, myopathy, tendinopathy or an inflammatory joint problem, BMI $>25 \mathrm{~kg} / \mathrm{m}^{2}$, any medication except birth controls pills in women, muscular trauma or simple fall in the preceding week, febrile illness in the preceding month. No physical activity was allowed in the week prior the investigation. Participants' characteristics are listed in table 1.

\section{Data collection}

After a detailed medical history and physical examination, participants' weight, height, blood pressure and body composition parameters were determined (Body Impedance Analyzer, Nutrigard-M, Frankfurt, Germany).

\section{Vibration exercise}

Our vibration platform consists of a plate oscillating around a middle axis, with feet placed on either side, resulting in a alternating up and down movement (Sismo Fitness Elite, Sismo Fitness International, 94230 Cachan, France). Vibration frequency range is from 1 to $32 \mathrm{~Hz}$, with an amplitude of $15 \mathrm{~mm}$. The exercise frequency was chosen at 26 $\mathrm{Hz}$, because our previous observation shows that it is well tolerated while providing a high muscular load. It has also been used in various other trials with oscillating platforms $[18,19]$.

The protocol intended to reproduce a difficult vibration training session that involves a large part of the body mus-

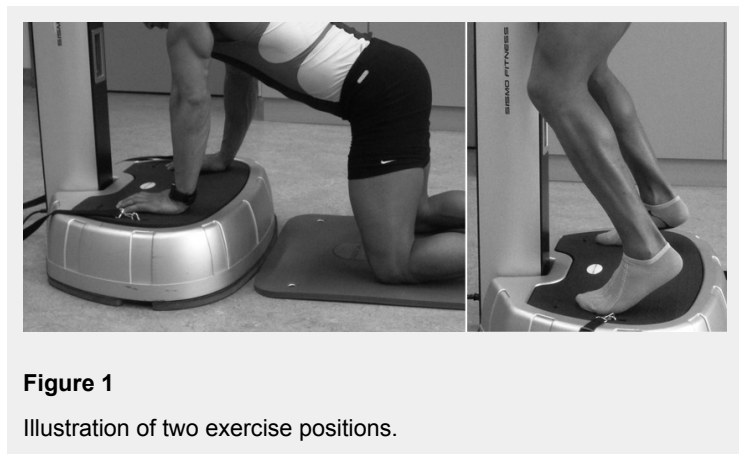

culature. First, three isometric exercises were executed, each one repeated three times, for a duration of ninety seconds, with forty seconds of rest in between. The exercises comprised a standing squat position with sixty degrees knee flexion, a standing on tip toes squat with thirty degrees knee flexion and a push up position with slightly bended elbows. Next, the sequence was repeated with dynamic exercises involving movement (squatting down or doing push ups). Feet and hands were placed at a constant distance from the rotating axis, corresponding to an oscillation amplitude of $15 \mathrm{~mm}$. Total exercise time was twenty seven minutes. This sequence is described in table 2. Two exercises are depicted in figure 1.

When standing, participants could hold the handles in front of them, but only to balance themselves, not to pull with the arms.

\section{Blood analysis}

A venous blood sample was taken prior to exercise, and the following parameters were determined: creatine-kinase (total and MB fraction), troponin-I, lactate dehydrogenase, aspartate aminotransferase. Repeat blood analysis was done at 24, 48 and 96 hours post exercise. Immediately after the exercise session, capillary lactate was analysed (Lactate-scout ${ }^{\circledR}$ portable analyzer, SensLab ${ }^{\circledR}$, Leipzig, Germany).

\section{Statistical analysis}

The time-course of CK blood levels was summarized for each subject as the area under curve (CK_AUC), calculated from $\mathrm{T} 0$ to $\mathrm{T} 4$ ( $\mathrm{T} 0=$ baseline value, $\mathrm{T} 4=$ day 4 or 96 hours post exercise), using the trapezoidal rule with appropriate subtraction of the value at baseline. In this way, a positive AUC would denote an increase in CK levels following the session on the vibration platform, whereas a zero or negative value would occur in absence of such an effect. The unilateral null hypothesis that the expected value of the AUC was zero or negative was tested with the Wilcoxon signed-rank test, across all subjects, as well as separately for women and men.

In addition, a comparison was made between men and women for all measured variables, using the bilateral form of the Mann-Whitney test for independent samples. The alpha level of all tests was 0.05 .

\section{Ethical considerations}

This study was approved by our institutional ethics committee and carried out in accordance with the Declaration of Helsinki. Written informed consent was obtained from each subject.

\section{Results}

The investigations were carried out between March and April 2009. All participants completed the study. One participant had a baseline CK elevation at $446 \mathrm{U} / 1$, which remained unexplained and asymptomatic. The $\mathrm{CK}$ response to exercise was highly variable. Five participants (25\%) showed a significant increase post exercise, defined as a maximal value of at least double of the baseline value. 
Maximal values were reached on the first day post exercise for one participant, and on the fourth day for the remaining four. The highest CK value reached $3520 \mathrm{U} / 1$ and was measured in a female participant at 96 hours. Figure 2 shows the CK evolution in all participants.

The CK_AUC was significantly higher than zero, irrespective of study group (median, interquartile range: 143, 33-485 IU*days, $p<0.005)$, women only $(113,30-7009$, $\mathrm{p}<0.01)$ or men only $(154,-10-506, \mathrm{p}<0.05)$, with a difference between genders that was not statistically significant $(\mathrm{p}>0.5)$.

The MB fraction of creatine kinase never increased significantly, neither did we observe a rise in troponin-I, except in the one subject who had a high baseline CK $(0.05$ at 24 hours). LDH did not increase after exercise. AST increased considerably at 96 hours in one participant (participant 01 , baseline of 20 to $91 \mathrm{U} / \mathrm{l}$ ), who also had the highest CK response. One other participant showed an increase in AST more than double baseline (participant 15, from 20 to $45 \mathrm{U} /$ 1).

Lactate values immediately post exercise showed a substantial elevation in all subjects, amounting to $10.0 \mathrm{mmol} / \mathrm{l}$ \pm 2.4 in men and $6.9 \pm 2.4$ in women, reflecting the intensity of the anaerobic muscular work (elevation was significantly more important in men compared to women, $\mathrm{p}=$ 0.009).

Delayed onset muscular soreness was present to some degree in all participants, but no correlation was found between its intensity and CK response.

\section{Discussion}

Our study confirms that common vibration exercises, as often executed by insufficiently active people in health centres or at home, can produce elevations in various markers of muscle damage. Creatine kinase elevation can be quite significant, reaching levels considered pathological in some cases. The reasons for the variability between participants is insufficiently explained and possibly involves a genetic component [20]. The participants were all similarly inactive and of a comparable fitness level according to questionnaires. Although it is known that intensive muscu- lar contractions, particularly of the eccentric-type (as when someone is resisting while lowering a weight, or walking downhill), can lead to the release of muscle enzymes, it has not been investigated in WBV, which is marketed as safe and easy to carry out. One female subject reached a value of $3520 \mathrm{U} / 1$ on the fourth day post-exercise, which is the habitual peaking time after eccentric contractions. The movement elicited by the platform is a form of alternating concentric and eccentric contractions (the person

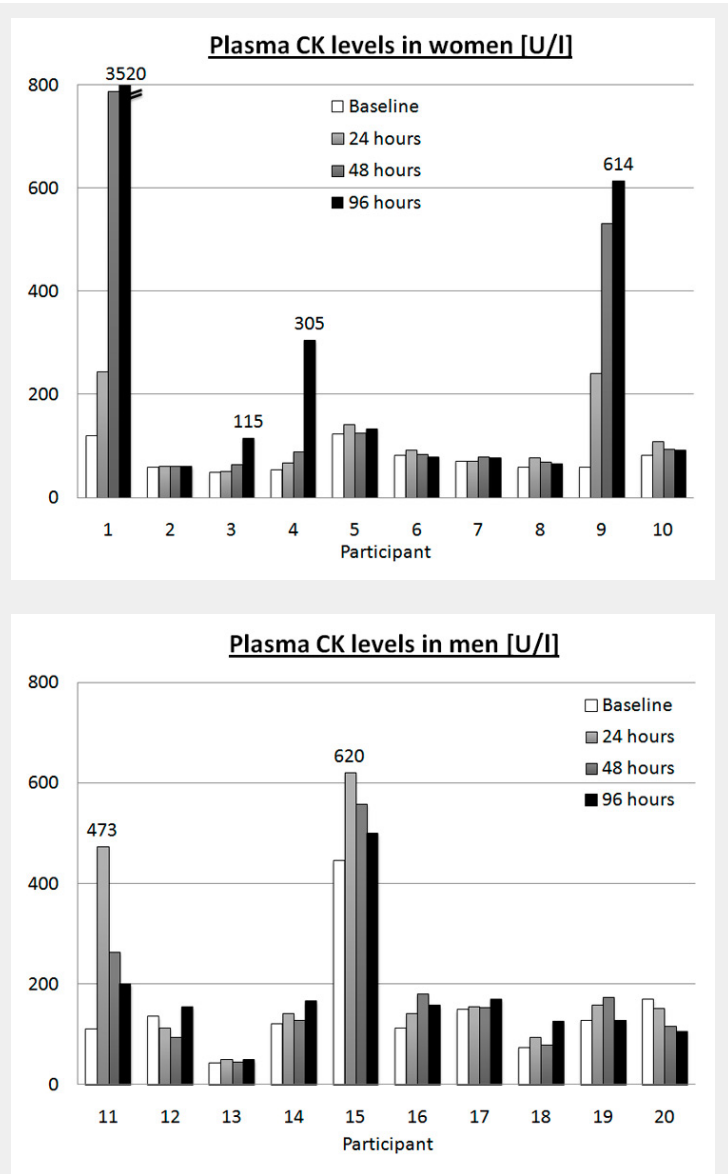

Figure 2

CK values in each participant at baseline, 24, 48 and 96 hours post exercise. Top: women, bottom: men.

\begin{tabular}{|l|l|l|l|}
\hline \multicolumn{2}{|l|}{ Table 1: Baseline characteristics of participants. } & Men $(\mathrm{n}=10)$ & p-value \\
\hline & Women $(\mathrm{n}=10)$ & $23.0 \pm 3.5$ & $\mathrm{~ns}$ \\
\hline Age & $22.4 \pm 1.4$ & $70.9 \pm 6.0$ & 0.005 \\
\hline Weight $(\mathrm{kg})$ & $62.0 \pm 5.0$ & $1.77 \pm 0.05$ & 0.006 \\
\hline Height $(\mathrm{m})$ & $1.68 \pm 0.06$ & $22.8 \pm 2.3$ & $\mathrm{~ns}$ \\
\hline BMl $\left(\mathrm{kg} / \mathrm{m}^{2}\right)$ & $22.1 \pm 1.9$ & $17.7 \pm 4.3$ & 0.0002 \\
\hline Body fat $(\%)$ & $27.6 \pm 3.1$ & $58.2 \pm 3.7$ & 0.0002 \\
\hline Fat-free mass $(\mathrm{kg})$ & $44.8 \pm 2.6$ & & \\
\hline & & & \\
\hline
\end{tabular}

\begin{tabular}{|c|c|c|c|c|}
\hline & & duration & pause & number of repetitions \\
\hline \multirow[t]{3}{*}{ isometric } & 1. Half squat (60 deg knee flexion) & 90 s & $40 s$ & 3 \\
\hline & 2. Tip-toed squat (30 deg knee flexion) & $90 \mathrm{~s}$ & $40 \mathrm{~s}$ & 3 \\
\hline & 3. Push-up position (30 deg elbow flexion) & $90 \mathrm{~s}$ & $40 \mathrm{~s}$ & 3 \\
\hline \multirow[t]{4}{*}{ dynamic } & 1. Squat to $90 \mathrm{deg}$ & $90 \mathrm{~s}$ & $40 \mathrm{~s}$ & 3 \\
\hline & 2. Squat on tip-toes & $90 \mathrm{~s}$ & $40 \mathrm{~s}$ & 3 \\
\hline & 3. Push-ups to 90 deg elbow flexion & $90 \mathrm{~s}$ & $40 \mathrm{~s}$ & 3 \\
\hline & & \multicolumn{3}{|c|}{ Total exercise duration: $270 \mathrm{~s} \times 6=\mathbf{2 7} \mathbf{~ m i n}$} \\
\hline
\end{tabular}


exercising shortens and stretches the muscle alternatively with each vibration cycle), which could account for this response. When the CK response is aggregated over the four days of the study period, it shows a significant elevation of the CK_AUC in all participants, but the variability of this response is highlighted by the significant interquartile ranges observed. The other blood enzymes measured showed very little variation, with no change for CK-MB fraction, LDH, ALT, or troponin-I. The only other parameter that was significantly increased (more than twice the baseline value) was AST, in two subjects. One was the female subject with the highest CK response. These changes are slight and difficult to interpret and altogether of little value, since AST can be easily modified by multiple factors (diet or alcohol consumption), although we asked our subjects to refrain from alcohol consumption for the duration of the study.

The widespread use of statins for the treatment and prevention of cardiovascular disease is linked with rare cases of severe rhabdomyolysis [21], and not so rare complaints of muscle aches or even mild myositis. Recently, the American College of Cardiology and American Heart Association (ACC/AHA) have defined four syndromes related to statin safety [22]: statin myopathy (any muscle complaints), myalgia (muscle complaints without CK elevation), myositis (with CK elevation) and rhabdomyolysis (CK elevation of more than 10 times the upper limit of normal). These definitions have limitations, and one of them is that they do not consider the patient with CK elevation and no muscle complaint. Most cases are found in patients on routine blood tests in the absence of symptoms, which can sometimes be a cause for concern due to confusion with myocardial damage, although the myocardial enzyme fraction is rarely elevated. Also, CK elevation can be magnified in treated patients post-exercise [23]. There has been a lot of concern with the side effects of such a widely prescribed drug, and treatment interruption because of muscle pain or CK elevation is probably not always indicated. In a review of randomized trials, Thompson et al. have found that the occurrence of these events are similar in control groups on placebo and in statin-treated patients [24]. Overall, it seems that the incidence of severe muscle complaints is not high, and concern is exaggerated. On the other hand, heavy exercise seems to potentially interact with (and add to) statin toxicity through a wide age range (18 to 65 years old), and practitioners should be aware of this phenomenon [23]. We have shown here that a common exercise modality such as WBV, adopted by a growing number of sedentary, maybe overweight persons, can cause various degrees of muscle damage shown by CK elevation. Owing to the prevalence of hypercholesterolaemia and the importance of statin treatment, the potential drug independent effect noted in the present study is of interest to the prescribing physician. Cessation of therapy could have long term deleterious effects, and the understanding that this reaction to exercise is often transient, with decreasing effect as the person becomes accustomed to exercise, should prompt physicians to pursue the treatment, and follow up on the symptoms and CK values. The debate on statin induced myopathy and its management is an often discussed issue, as millions of per- sons are treated each year. Joy and Hegele have recently reviewed this topic [25].

A second point which should be highlighted is the high anaerobic component of WBV exercises, reflected by a high level of lactate (comparable to maximal aerobic exercise testing). Our protocol was designed to elicit an elevated muscular load, but it resembles those commonly carried out by the naïve user.

In a similarly designed study (unpublished, currently in review) aimed at comparing the metabolic demand of WBV exercises compared to the same exercises without vibration, we have observed that heart rate is stimulated at $69.8 \%$ of the maximal capacity (and $32.4 \%$ of maximal oxygen uptake or $\mathrm{VO} 2_{\max }$ ) on average for the whole session, but peaks at $80.2 \%$ ( $45 \%$ for $\left.\mathrm{VO} 2_{\max }\right)$ for the most difficult exercises, which is habitually associated with moderate to vigorous health enhancing physical activity, but barely sufficient to elevate lactate. This happens only when a major part of the exercise is of anaerobic nature, as in intensive resistance training. The safety of such intense exercise is not guaranteed in the presence of comorbidities such as diabetes, renal or liver failure, or coronary heart disease, as it could lead to unduly metabolic stress.

The weaknesses in our study include a small number of participants and a high variation of muscle damage response. In addition, we have not examined the evolution of CK response after repetition of the same protocol on various occasions. And finally we must acknowledge the fact that our subjects are younger than the habitual patients taking statins, but muscle enzymes response to exercise has been shown to be similar in young and middle aged subjects (18 to 65 years old) [23]. Nevertheless we believe that our data are compelling enough and provide the general practitioner with insight on a novel exercise modality.

In conclusion, the link between physical inactivity and overall or cardiovascular mortality is well established. Most of the studies put emphasis on cardiovascular fitness. However, a higher level of muscular strength is associated with reduced overall mortality, independently of aerobic fitness [26]. WBV provides both types of exercise, potentially increasing cardiovascular fitness and muscular strength [10]. Awareness that WBV can mimic the side-effects of statins is of interest to practitioners, who should definitely have these considerations in mind before discontinuing medication because of muscle pain or muscle enzyme elevation.

We acknowledge the contribution of Chantal Daucourt for help in the collection of blood samples.

Funding / potential competing interests: This study was funded by departmental funds from the Clinical Pathophysiology Division. No funding was obtained from outside sources. All authors received no financial support for the submitted work from anyone other than their employer, have no financial relationships with commercial entities that might have an interest in the submitted work and no non-financial interests that may be relevant to the submitted work. 
Correspondence: Dr. Boris Gojanovic, MD, Sports

Medicine Unit, CHUV, Pierre Decker 4, CH-1011

Lausanne,Switzerland, boris.gojanovic@chuv.ch

\section{References}

1 Rehn B, et al. Effects on leg muscular performance from whole-body vibration exercise: a systematic review. Scand J Med Sci Sports. 2007;17(1):2-11

2 Gusi N, Raimundo A, Leal A. Low-frequency vibratory exercise reduces the risk of bone fracture more than walking: a randomized controlled trial. BMC Musculoskelet Disord. 2006;7:92.

3 Bautmans I, et al. The feasibility of Whole Body Vibration in institutionalised elderly persons and its influence on muscle performance, balance and mobility: a randomised controlled trial [ISRCTN62535013]. BMC Geriatr. 2005;5:17.

4 Bruyere $\mathrm{O}$, et al. Controlled whole body vibration to decrease fall risk and improve health-related quality of life of nursing home residents. Arch Phys Med Rehabil. 2005;86(2):303-7.

5 Cheung WH, et al. High-frequency whole-body vibration improves balancing ability in elderly women. Arch Phys Med Rehabil. 2007;88(7):852-7.

6 Runge M, Rehfeld G, Resnicek E. Balance training and exercise in geriatric patients. J Musculoskelet Neuronal Interact. 2000;1(1):61-5.

7 Goto K, Takamatsu K. Hormone and lipolytic responses to whole body vibration in young men. Jpn J Physiol. 2005;55(5):279-84.

8 Baum K, Votteler T, Schiab J. Efficiency of vibration exercise for glycaemic control in type 2 diabetes patients. Int $\mathrm{J}$ Med Sci. 2007;4(3):159-63.

9 Bosco C, et al. Hormonal responses to whole-body vibration in men. Eur J Appl Physiol. 2000;81(6):449-54.

10 Bogaerts AC, et al. Effects of whole body vibration training on cardiorespiratory fitness and muscle strength in older individuals (a 1-year randomised controlled trial). Age Ageing. 2009;38(4):448-54.

11 Myers J, et al. Exercise capacity and mortality among men referred for exercise testing. N Engl J Med. 2002;346(11):793-801.
12 Sesso HD, Paffenbarger RS Jr, Lee IM. Physical activity and coronary heart disease in men: The Harvard Alumni Health Study. Circulation. 2000;102(9):975-80.

13 Warburton DE, Nicol CW, Bredin SS. Health benefits of physical activity: the evidence. CMAJ. 2006;174(6):801-9.

14 Clarkson PM, Hubal MJ. Exercise-induced muscle damage in humans. Am J Phys Med Rehabil. 2002;81(11Suppl):S52-69.

15 Suzuki K, et al. Changes in markers of muscle damage, inflammation and HSP70 after an Ironman Triathlon race. Eur J Appl Physiol. 2006;98(6):525-34.

16 Clarkson PM, et al. Serum creatine kinase levels and renal function measures in exertional muscle damage. Med Sci Sports Exerc. 2006;38(4):623-7.

17 International Physical Activity Questionnaire. [cited 2010 July 6th]; Available from: http://www.ipaq.ki.se/ipaq.htm.

18 Rees SS, Murphy AJ, Watsford ML. Effects of whole-body vibration exercise on lower-extremity muscle strength and power in an older population: a randomized clinical trial. Phys Ther. 2008;88(4):462-70.

19 Rees S, Murphy A, Watsford M. Effects of vibration exercise on muscle performance and mobility in an older population. J Aging Phys Act. 2007;15(4):367-81.

20 Clarkson PM, et al. ACTN3 and MLCK genotype associations with exertional muscle damage. J Appl Physiol. 2005;99(2):564-9.

21 Staffa JA, Chang J, Green L. Cerivastatin and reports of fatal rhabdomyolysis. N Engl J Med. 2002;346(7):539-40.

22 Pasternak RC, et al. ACC/AHA/NHLBI clinical advisory on the use and safety of statins. J Am Coll Cardiol. 2002;40(3):567-72.

23 Thompson PD, et al. Lovastatin increases exercise-induced skeletal muscle injury. Metabolism. 1997;46(10):1206-10.

24 Thompson PD, Clarkson P, Karas RH. Statin-associated myopathy. JAMA. 2003;289(13):1681-90.

25 Joy TR, Hegele RA. Narrative review: statin-related myopathy. Ann Intern Med. 2009;150(12):858-68.

26 Ruiz JR, et al. Muscular strength and adiposity as predictors of adulthood cancer mortality in men. Cancer Epidemiol Biomarkers Prev. 2009;18(5):1468-76. 